\title{
VALORES HEMATOLÓGICOS Y BIOQUÍMICOS DE JUVENILES DE PAICHE Arapaima gigas EN CULTIVO INTENSIVO
}

\author{
Anai GONZALES ${ }^{1}$, Félix MEJÍA ${ }^{1}$, Katty HUANUIRI ${ }^{1}$, Ingrid SÁNCHEZ ${ }^{1,2}$, Jorge VÁSQUEZ ${ }^{1}$, Christian \\ FERNÁNDEZ-MÉNDEZ 1 \\ 1 Instituto de Investigaciones de la Amazonia Peruana-IIAP, Programa para el Uso y Conservación del Agua y sus Recursos- \\ AQUAREC, Carretera Iquitos Nauta km 4,5, Apartado 784, Iquitos, Perú. E-mail: cfernandez@iiap.org.pe \\ 2 Universidad Nacional de Colombia, Facultad de Medicina Veterinaria y de Zootecnia, Departamento de producción \\ animal, Bogotá, Colombia.
}

\begin{abstract}
RESUMEN
El objetivo de este estudio fue determinar los valores hematológicos y bioquímicos de juveniles de paiche Arapaima gigas criados en cultivo intensivo. Los juveniles (peso inicial 71,72 $\pm 23,89 \mathrm{~g}$ y longitud inicial $20,45 \pm 1,42 \mathrm{~cm}$ ) fueron estabulados en cuatro tanques de $900 \mathrm{~L}$ con recirculación de agua (temperatura 25,90 $\pm 0,44^{\circ} \mathrm{C}$; oxígeno disuelto $1,43 \pm 1,12 \mathrm{mg} \mathrm{L}^{-1} ; \mathrm{pH} 6,74 \pm 0,2 ;$ nitrito $0,41 \pm 0,62 \mathrm{mg} \mathrm{L}^{-1}$ y amonio $16,17 \pm 9,03$ $\mathrm{mg} \mathrm{L}^{-1}$ ) por un periodo de 154 días. Durante los primeros 40 días, se mantuvieron a una densidad inicial de 200 peces $\mathrm{m}^{-3}$ y posteriormente a 150 peces $\mathrm{m}^{-3}$. La alimentación se realizó tres veces al día con un alimento balanceado (Aquatech ${ }^{\circledR}$, Naltech, Lima, Perú; composición proximal: 42\% proteína, 10\% lípidos, 12\% cenizas; tamaño de partícula: 2,5 y $4 \mathrm{~mm}$ ). Las muestras de sangre se obtuvieron en cinco muestreos (124, $137,138,142$ y 143 días) con jeringas descartables conteniendo EDTA al 10\% para la determinación de los valores hematológicos y bioquímicos. Al final del experimento, los peces alcanzaron un peso promedio de $832,6 \pm 173,1 \mathrm{~g}$ y $47,4 \pm 2,4 \mathrm{~cm}$ de longitud total con una supervivencia de $91,9 \%$. Los valores hematológicos y bioquímicos promedio fueron: hematocrito (Hto) $32,2 \pm 1,4 \%$; leucocitos (Leu) $5,2 \pm 2,42 \times 10^{3} \mu \mathrm{L}^{-1}$; eritrocitos (Er) 1,3 $\pm 0,21 \times 10^{6} \mu \mathrm{L}^{-1}$; hemoglobina (Hb) $10,4 \pm 0,90 \mathrm{~g} \mathrm{dL}^{-1}$; volumen corpuscular medio (VCM) 249,9 \pm 40,2 fL; concentración de hemoglobina corpuscular media (CHCM) 32,3 $\pm 2,5 \mathrm{~g} \mathrm{dL}^{-1}$; hemoglobina corpuscular media (HCM) 80,6 $\pm 13,8 \mathrm{~g} \mathrm{dL}^{-1}$; albúmina $2,26 \pm 0,82 \mathrm{~g} \mathrm{dL}^{-1}$; glucosa $54,83 \pm 43,09 \mathrm{mg} \mathrm{dL}^{-1} \mathrm{y}$ colesterol 104,63 $\pm 43,89 \mathrm{mg} \mathrm{dL}^{-1}$. Los valores hematológicos y bioquímicos se encontraron dentro de los rangos reportados para esta especie, mostrando condiciones fisiológicas normales al estar sometidos a condiciones intensivas de cultivo.
\end{abstract}

PALABRAS CLAVE: Amazonia, acuicultura, piscicultura, paiche.

\section{HEMATOLOGICAL AND BIOCHEMICAL VALUES FOR Arapaima gigas JUVENILES UNDER INTENSIVE REARING CONDITIONS}

\begin{abstract}
The aim of this study was to determine hematological and biochemical values of juveniles of Arapaima gigas reared in intensive culture. Juveniles (initial wet weight and length: 71,72 $\pm 23,89 \mathrm{~g}$ and 20,45 $\pm 1,42$ $\mathrm{cm}$ ) were reared in four $900 \mathrm{~L}$ tanks with water recirculation (temperature $25,9 \pm 0.44{ }^{\circ} \mathrm{C}$, dissolved oxygen $1,43 \pm 1,12 \mathrm{mg} \mathrm{L}^{-1}, \mathrm{pH} 6,74 \pm 0,2$, nitrite $0,41 \pm 0,62 \mathrm{mg} \mathrm{L}^{-1}$ and ammonium $16,17 \pm 9,03 \mathrm{mg} \mathrm{L}^{-1}$ ) for a period of 154 days. During the first 40 days, they were maintained at an initial density of $200 \mathrm{~m}^{-3}$ fish and at $150 \mathrm{~m}^{-3}$ fish later. Feeding was done three times a day with a compound diet (Aquatech ${ }^{\circledR}$, Naltech, Lima, Peru; proximate composition: $42 \%$ proteins, $10 \%$ lipids, $12 \%$ ash; particle size: $2,5 \mathrm{y} 4 \mathrm{~mm}$ )). Blood samples were obtained in five samples $(124,137,138,142$ y 143 days) with syringes containing 10\% EDTA for the determination of hematological and biochemical values. At the end of the experiment, the fish reached an average weight of $832,6 \pm 173,1 \mathrm{~g}$ and $47,4 \pm 2,4 \mathrm{~cm}$ of total length, and $91,9 \%$ of survival. The mean hematological and biochemical values were: hematocrit (Hto) $32,2 \pm 1,4 \%$; leukocytes (Leu) $5,2 \pm 2,22 \times 10^{3} \mu \mathrm{L}^{-1}$; erythrocytes (Er) $1,3 \pm 0,21 \times 10^{6} \mu \mathrm{L}^{-1}$; hemoglobin (Hb) 10,4 $\pm 0,90 \mathrm{~g} \mathrm{dL}^{-1}$; mean corpuscular volume (MCV) 249,9 $\pm 40,2$ $\mathrm{fL}$; mean corpuscular hemoglobin concentration (MCHC) $32,3 \pm 2,5 \mathrm{~g} \mathrm{dL}^{-1}$; mean corpuscular hemoglobin (MCH) 80,6 $\pm 13,8 \mathrm{~g} \mathrm{dL}^{-1}$; albumin 2,26 $\pm 0,82 \mathrm{~g} \mathrm{dL}^{-1}$; glucose 54,83 $\pm 43,09 \mathrm{mg} \mathrm{dL}^{-1}$ and cholesterol 104,63 \pm $43,89 \mathrm{mg} \mathrm{dL}^{-1}$. Hematological and biochemical values were within the ranges reported for this species, showing normal physiological conditions when subjected to intensive culture conditions.
\end{abstract}

KEYWORDS: Amazon, aquaculture, fish farming, paiche. 


\section{INTRODUCCIÓN}

El paiche, Arapaima gigas, es un pez piscívoro que habita en las regiones amazónicas y sus afluentes (Lüling, 1971), puede llegar a medir hasta 3 metros de longitud (Nelson, 1994) y se caracteriza por presentar respiración aérea obligatoria (SaintPaul, 1986). Desde hace décadas esta especie ha venido soportando una explotación pesquera intensa debido a la demanda de su carne y por la exportación de alevinos como pez ornamental (Saint-Paul \& Bayley, 1979; García et al., 2011). Por lo tanto, es considerada como especie amenazada en el Apéndice II de la lista CITES, por lo que su comercio internacional se regula mediante procedimientos de extracción del medio natural no perjudicial (Castello \& Stewart, 2010). A raíz de esta demanda y sobrepesca, ha surgido el interés por la producción de $A$. gigas en ambientes controlados (Cavero et al., 2003a; Cavero et al., 2003b; PereiraFilho et al., 2003; Del Risco et al., 2008; Barrera \& Almeyra, 2010). Esta especie se reproduce en cautiverio (Alcántara, 1990; Nuñez et al., 2011) y acepta la alimentación con dietas balanceadas (Velásquez et al., 2007), presentando un rápido crecimiento, alcanzando un peso en torno a $10 \mathrm{~kg}$ en un año de cultivo (Imbiriba, 2001). Puede tolerar altas densidades y poco volumen de agua (Cavero et al., 2003b) con bajos niveles de oxígeno disuelto y altos niveles de amonio (Cavero et al., 2004) debido a su respiración aérea obligatoria, obteniendo mayor parte de oxígeno a través de la vejiga natatoria (Brauner \& Val, 1996). Hasta el momento poco se conoce sobre los parámetros hematológicos y bioquímicos de esta especie en cultivo intensivo. El análisis de los parámetros sanguíneos y bioquímicos son una herramienta útil para el diagnóstico y pronóstico de las patologías que son más frecuentes a medida que se intensifican los sistemas de cultivo, ya que todas estas condiciones determinan una serie de alteraciones en la sangre (Ranzani-Paiva et al., 2013). De esta manera el objetivo del presente estudio fue determinar los valores hematológicos y bioquímicos de juveniles de paiche criados en condiciones de cultivo intensivo.

\section{MATERIALES Y MÉTODOS}

\section{CONDICIONES DE CULTIVO}

Se utilizaron un total de 580 juveniles de paiche con un peso inicial de $71,72 \pm 23,89 \mathrm{~g}$ y una longitud total de $20,43 \pm 1,42 \mathrm{~cm}$ cultivados en cuatro tanques de concreto de 900 litros cada uno con filtración mecánica y recirculación de agua durante 154 días en las instalaciones del Centro de Investigaciones Fernando Alcántara Bocanegra (CIFAB) del
Instituto de Investigaciones de la Amazonia Peruana (IIAP). Los peces fueron alimentados ad libitum con una dieta comercial extruida $\left(\right.$ Aquatech $^{\circledR}$, Naltech, Lima, Peru; composición proximal: $42 \%$ proteína, $10 \%$ lípidos, $12 \%$ cenizas; $1,5 \%$ de calcio; $1 \%$ de fosforo; tamaño de particula: 2,5 y $4 \mathrm{~mm}$ ) a una frecuencia de tres veces por día ( 8,12 y 16 horas). Durante los primeros 40 días se mantuvieron a una densidad de 200 peces $\mathrm{m}^{-3} \mathrm{y}$ durante el resto del experimento a 150 peces $\mathrm{m}^{-3}$.

Las mediciones de los parámetros físicos y químicos del agua (temperatura, oxígeno disuelto y $\mathrm{pH}$ ) se realizaron dos veces al día (7 y 16 horas) con un oxímetro (YSI, Model 55) y un medidor de $\mathrm{pH}$ (Oakton, 110 series). El amoniaco y los nitritos se evaluaron dos veces por semana mediante un fotómetro colorimétrico (Hanna Instruments, HI 83203).

Para los cálculos de la tasa de crecimiento especifico (TCE) se consideró: $\mathrm{TCE}=[\ln$ (peso final) - $\ln$ (peso inicial)]/diasx100; la ganancia de peso diario (GPD) $: \mathrm{GDP}=($ peso final - peso inicial $) /$ días $\mathrm{y}$ tasa de producción $=($ biomasa final $/$ volumen de agua).

\section{COLECTA DE SANGRE Y ANÁLISIS}

Los peces fueron colectados con una red de mano y ubicados en una esponja, se emplearon toallas húmedas para la inmovilización y vendaje de los ojos, hidratando constantemente el cuerpo y las branquias con agua. Se extrajo $1 \mathrm{ml}$ de sangre mediante punción de la arteria caudal a nivel del arco hemal, utilizando jeringas desechables conteniendo EDTA al 10\%; la sangre extraída fue separada en dos partes: para muestras hematológicas se colocó la sangre en tubos de $2 \mathrm{ml}$ conservadas en refrigeración $\left(4{ }^{\circ} \mathrm{C}\right)$ para preservar la viabilidad de las células y para las muestras bioquímicas en tubos de $0,5 \mathrm{ml}$ que fueron centrifugados a $100 \mathrm{rpm}$ para obtener el plasma sanguíneo.

Las muestras de sangre colectadas fueron usadas en la determinación de hematocrito (Hto) por el método del microhematocrito (Goldenforb et al., 1971), de la concentración de hemoglobina $(\mathrm{Hb})$ por el método de la cianometahemoglobina (Laboratorios BIOLABTEST ${ }^{\circledR}$ ), del conteo de eritrocitos (Er) y leucocitos (Leu) con solución Natt \& Harrick (1952) a través de cámara de Neubauer, de la determinación de los índices hematimétricos: volumen corpuscular medio (VCM), concentración de hemoglobina corpuscular media (CHCM) y hemoglobina corpuscular media (HCM) según el método de Wintrobe (1934). Para realizar el recuento diferencial de leucocitos se realizaron frotis sanguíneos teñidos con reactivo Wright por 10 
minutos (Hawkey y Dennett, 1989). En cuanto a los parámetros bioquímicos, se determinó la concentración de albumina (método colorimetrico: Proti2, Laboratorios Wiener ${ }^{\circledR}$ ), de glucosa (método Trinder: Laboratorios FAR Diagnostics $($ ) y de colesterol (método Trinder: Laboratorios FAR Diagnostics $\left.{ }^{\circledR}\right)$. Las lecturas fueron realizadas en un espectrofotómetro de UV visible (Agilent Technologies, Cary 60. California, Estados Unidos) .

\section{ANÁLISIS DE DATOS}

Los datos se analizaron mediante estadística descriptiva, considerando el promedio, la desviación estándar, los valores mínimo y máximo y el coeficiente de variación. Para determinar la correlación entre variables se usó el coeficiente de Pearson con el software estadístico "Sigma Plot 11".

\section{RESULTADOS Y DISCUSIÓN}

Los valores de los parámetros físicos y químicos del agua de los tanques donde se cultivaron los juveniles de A. gigas se muestran en la Tabla 1. La temperatura, el $\mathrm{pH}$ y el nitrito estuvieron dentro de los valores normales para el cultivo de paiche en condiciones de cautiverio (Alcántara \& Guerra, 1992; Cavero et al., 2003b). Sin embargo, el valor promedio de oxígeno disuelto fue bajo y de amonio alto en comparación con otros estudios (Alcántara \& Guerra, 1992; Cavero et al., 2003b; Paredes et al., 2013). En los sistemas acuícolas intensivos, la concentración de oxigeno disminuye debido a la gran demanda ocasionada por las altas densidades de peces y al alimento, que constituye la principal fuente de compuestos nitrogenados en el agua y que aumenta en el caso de peces carnívoros por los elevados niveles de proteína usados en las raciones en comparación con peces omnívoros (Pereira \& Mercante, 2005; Ismiño-Orbe et al., 2003). Sin embargo, esta especie tiene una ventaja respecto a los peces de respiración acuática (Cavero et al., 2003b) por su respiración aérea obligatoria, obteniendo mayor parte de oxígeno a través de la vejiga natatoria (Brauner \& Val, 1996), lo que le permite tolerar aguas con bajos niveles de oxígeno disuelto y altos niveles de amonio (Cavero et al., 2004).

A los 154 días de cultivo con una densidad de 150 a 200 peces $\mathrm{m}^{-3}$ los peces alcanzaron un peso de $832,6 \pm 173,1 \mathrm{~g}$ y una longitud total de $47,4 \pm 2,4 \mathrm{~cm}$. La curva de crecimiento se muestra en la Figura 1. Varios estudios con $A$. gigas a menores densidades $\left(10 \mathrm{y} 12,5\right.$ peces $\left.\mathrm{m}^{-3}\right)$ muestran un peso promedio final de 2630,4 y $2138 \mathrm{~g}$ respectivamente en 140 días de cultivo (De Oliveira et al., 2012), mientras que a una densidad de 22 peces $\mathrm{m}^{-3}$ en 200 días de cultivo se obtuvo $1172 \mathrm{~g}$ (Cavero et al., 2003). Estos resultados son superiores a los obtenidos en el presente estudio debido principalmente a la menor densidad, que brinda una ventaja de crecimiento. En la Figura 2 se proporciona una relación entre la longitud total (LT) $\mathrm{y}$ el peso (P), calculada a partir de todos los individuos.

La sobrevivencia final durante los 154 días de cultivo fue del 91,9\%. Resultados de supervivencia del 91,7 a $100 \%$ son reportados para el cultivo de $A$. gigas (Pereira-Filho et al., 2003; Menezes et al., 2006; De Oliveira et al., 2012). La mortalidad de peces fue mayormente debido a infecciones por hongos y bacterias en heridas de la aleta caudal ocasionadas por mordeduras relacionadas a la alta densidad de cultivo. La TCE fue de $1,59 \pm 0,03 \%$ dia ${ }^{1}$. Algunos estudios muestran en esta misma especie valores superiores, de $2,22-2,25 \% \mathrm{dia}^{-1}$ cultivados durante 140 días (De Oliveira et al., 2012) y otros inferiores, de $1,09 \%$ dia $^{-1}$ cultivados durante 365 días (Pereira-Filho et al., 2003). La GPD encontrada fue de 4,94 $\pm 0,25 \mathrm{~g} \mathrm{dia}^{-1}$ en contraste con los $14,6-18 \mathrm{~g}$ dia $^{-1}$ que obtuvo De Oliveira et al. (2012) y los $18.9 \mathrm{~g}$ $\operatorname{dia}^{-1}$ de Pereira-Filho et al. (2003). Sin embargo, la tasa de producción obtenida en el presente estudio de $105.3 \pm 4,3 \mathrm{~kg} \mathrm{~m}^{-3}$ fue superior a los $25,4-26,3 \mathrm{~kg} \mathrm{~m}^{-3}$

Tabla 1. Valores de los parámetros físicos y químicos del agua durante los 154 días de cultivo intensivo de $A$. gigas.

\begin{tabular}{lccc}
\hline \multicolumn{1}{c}{ Parámetros } & Promedio \pm DE & Mínimo & Máximo \\
\hline Temperatura $\left({ }^{\circ} \mathrm{C}\right)$ & $25,9 \pm 0,44$ & 25 & 27,3 \\
Oxígeno disuelto $\left(\mathrm{mg} \mathrm{L}^{-1}\right)$ & $1,43 \pm 1,12$ & 0,1 & 3,7 \\
$\mathrm{pH}$ & $6,74 \pm 0,20$ & 6,4 & 7,3 \\
Nitrito $\left(\mathrm{mg} \mathrm{L}^{-1}\right)$ & $0,41 \pm 0,62$ & 0,05 & 2,7 \\
Amonio $\left(\mathrm{mg} \mathrm{L}^{-1}\right)$ & $16,17 \pm 9,03$ & 4,9 & 37,9 \\
\hline
\end{tabular}



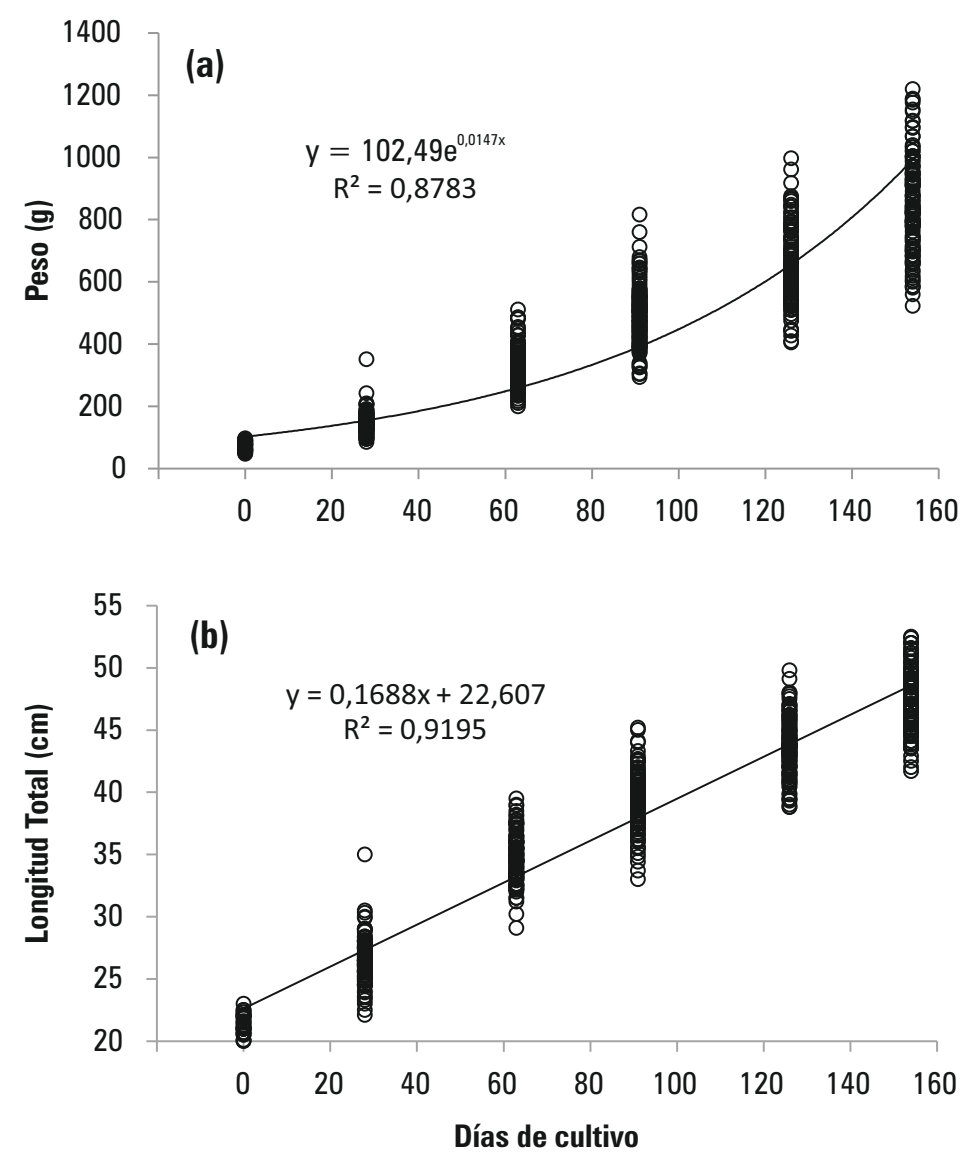

Figura 1. Peso (a) y longitud total (b) de A. gigas durante los 154 días de cultivo intensivo.

reportados por De Oliveira et al. (2012). Estos resultados muestran que a una mayor densidad, de $150-200$ peces $\mathrm{L}^{-1}$, a pesar de obtener un menor TCE y GPD, se consigue obtener mayor cantidad de carne por $\mathrm{m}^{3}$.

Los parámetros hematológicos son considerados buenos indicadores fisiológicos frente a una respuesta a estrés en peces, por transporte, captura, parámetros medioambientales y sobrepoblación, las cuales pueden alterar el patrón característico del eritrograma en teleósteos dulceacuícolas y marinos (Tavares-Días \& Moraes, 2004). En el promedio de los valores hematológicos (hematocrito, hemoglobina, valores de VCM, CHCM y HCM) se observó el aumento de algunos parámetros (Tabla 2) en comparación con otros trabajos para la misma especie (Paredes et al., 2013; Drumond et al., 2010; Serrano et al., 2013). Sin embargo estos valores se encuentran dentro de los valores normales reportados para juveniles de paiche en ambientes controlados (Tavares-Días et al., 2007; Menezes et al., 2006; Serrano et al., 2013) y de teleósteos dulceacuícolas mantenidos en cautiverio y del medio natural (Tavares-Días \& Moraes, 2004), a excepción del VCM, que se encontró ligeramente superior a lo reportado por los demás estudios. En este contexto, la variación de estos parámetros hematológicos en el presente estudio puede estar influenciado por los niveles de oxígeno disuelto ( 0.1 $-3,7 \mathrm{mg} \mathrm{L}^{-1}$ ) reportados, pues según Val et al. (1990), los peces se adaptan a bajos niveles de oxígeno incrementando los porcentajes de hematocrito y concentración de hemoglobina como estrategia para captar más eficientemente el poco gas disponible en el agua y lidiar con las condiciones de hipoxia.

Los leucocitos son células nucleadas de diferentes formas y funciones, entre los cuales se encuentran los linfocitos, neutrófilos, monocitos, eosinofilos y basófilos, que son usualmente observados en la sangre de los peces. Los linfocitos son los responsables en el reconocimiento de antígenos, los neutrófilos actúan contra infecciones 
bacterianas realizando fagocitosis, los monocitos actúan durante un proceso inflamatorio, los eosinofilos participan en procesos de defensa contra parásitos y los basófilos actúan en un proceso de fagocitosis (Ranzani-Paiva et al., 2013; Martins et $a l .$, 2004). En el presente trabajo, las extensiones sanguíneas evidenciaron cuatro leucocitos distintos: linfocitos, monocitos, eosinofilos y neutrófilos, habiendo mayor frecuencia de linfocitos en comparación con las demás células (Tabla 2).

Otros estudios con la misma especie mostraron un mayor porcentaje de linfocitos y monocitos (Tavares-Días et al., 2007; Drumond et al., 2010). No se observó la presencia de basófilos, concordando con otros reportes sobre $A$. gigas (Tavares-Días et al., 2007; Drumond et al., 2010; Marinho et al., 2015).

Los basófilos son leucocitos poco frecuentes en la sangre de peces y su ausencia desde hace mucho tiempo está relacionada con uso de técnicas de coloración inadecuadas en las extensiones sanguíneas. Los gránulos metacromáticos de los basófilos frecuentemente se disuelven a causa de fijadores de alcohol solubilizado, compuesto que está presente en la solución Wrigth (Campbell, 2004), dificultando su identificación en microscopia de luz (Tavares-Días \& Moraes, 2004; Tavares-Días, 2006).
Los niveles de albumina, colesterol y glucosa en A. gigas fueron inferiores a los valores reportados por Tavares-Días et al. (2007), pero superiores a lo reportado por Paredes et al. (2013), a excepción del colesterol. Estas variaciones en el mismo grupo etario se debe posiblemente a la variación de factores ambientales y no ambientales como alimentación, hábitos, movilización y modo de vida de los peces (Tavares-Días \& Moraes, 2004). Al parecer, la glucosa puede verse afectada por el estrés a causa de altos niveles de amonio (Cavero et al., 2004) lo que explicaría el aumento de este parámetro hematológico en el trabajo en comparación con los resultados obtenidos por Paredes et al. (2013). El colesterol, por su parte, influye directamente en el metabolismo, el transporte de lípidos y la actividad cardiovascular, además de ser un precursor de la formación de hormonas esteroides y los ácidos biliares importantes en la digestión y absorción de los lípidos en la dieta. Así mismo, el colesterol puede aumentar (Stickney, 2010) conforme se incrementa el nivel proteico en la ración (De Medeiros, 2014).

Los valores hematológicos y bioquímicos de los juveniles de A. gigas sometidas a condiciones intensivas de cultivo se encontraron dentro de los rangos reportados para esta especie, presentando ligeras variaciones, mostrando condiciones fisiológicas normales descritas para teleósteos.

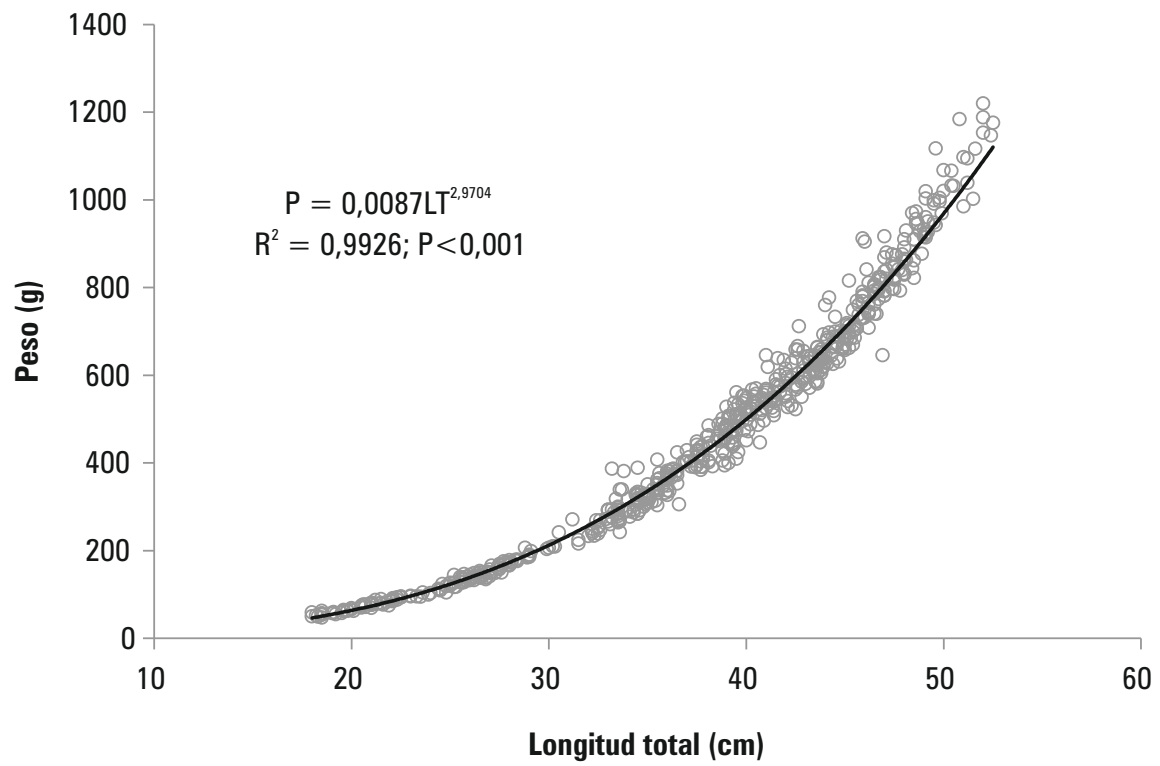

Figura 2. Correlación entre longitud total (LT) y peso (P) de $A$. gigas durante los 154 días de cultivo intensivo $(n=619)$ 
Tabla 2. Valores hematológicos, bioquímicos y porcentaje de células de defensa orgánica de $A$. gigas en cultivo intensivo.

\begin{tabular}{|c|c|c|c|c|}
\hline Parámetros & Promedio $\pm \mathrm{DE}$ & Mínimo & Máximo & CV (\%) \\
\hline Hematocrito (\%) & $32,2 \pm 1,4$ & 28,6 & 35,2 & 22,1 \\
\hline Hemoglobina $\left(\mathrm{g} \mathrm{dL}^{-1}\right)$ & $10,4 \pm 0,90$ & 8,5 & 12,7 & 11,5 \\
\hline Eritrocitos $\left(\times 10^{6} \mu \mathrm{L}^{-1}\right)$ & $1,3 \pm 0,21$ & 1,0 & 1,7 & 6,1 \\
\hline Leucocito $\left(x 10^{3} \mu \mathrm{L}^{-1}\right)$ & $5,2 \pm 2,42$ & 2,0 & 13,4 & 2,1 \\
\hline VCM (fL) & $249,9 \pm 40,2$ & 188 & 331,1 & 6,2 \\
\hline CHCM $\left(\mathrm{g} \mathrm{dL}^{-1}\right)$ & $32,3 \pm 2,5$ & 31,4 & 33,9 & 12,8 \\
\hline $\mathrm{HCM}\left(\mathrm{g} \mathrm{dL}^{-1}\right)$ & $80,6 \pm 13,8$ & 59,1 & 112,4 & 5,8 \\
\hline Linfocitos (\%) & $78,0 \pm 5,5$ & 65,0 & 90,0 & 15,0 \\
\hline Monocitos (\%) & $11,0 \pm 3,7$ & 3,0 & 20,0 & 2,7 \\
\hline Eosinófilos (\%) & $8,0 \pm 3,5$ & 3,0 & 10,0 & 2,4 \\
\hline Neutrófilos (\%) & $3,0 \pm 3,1$ & 3,0 & 10,0 & 1,0 \\
\hline Albumina $\left(\mathrm{g} \mathrm{dL}^{-1}\right)$ & $2,2 \pm 0,82$ & 0,10 & 4,9 & 2,7 \\
\hline Colesterol (mg dL $\left.{ }^{-1}\right)$ & $125,8 \pm 18,2$ & 91,9 & 161,7 & 2,3 \\
\hline Glucosa (mg dL $\left.{ }^{-1}\right)$ & $56,1 \pm 9,6$ & 22,7 & 82,6 & 1,2 \\
\hline
\end{tabular}

$\mathrm{VCM}=$ Volumen corpuscular medio, $\mathrm{HCM}=$ Concentración de hemoglobina y $\mathrm{CHCM}=$ Concentración de hemoglobina corpuscular media, $\mathrm{CV}=$ Coeficiente de variación.

\section{BIBLIOGRAFÍAS CITADAS}

Alcántara-Bocanegra, F.A. 2006. Observaciones sobre el comportamiento reproductivo del paiche, Arapaima gigas, en cautiverio. Folia Amazónica, 2(1-2): 163-166.

Alcántara-Bocanegra, F.A.; Guerra-Flores, H. 2006. Cultivo de paiche, Arapaima gigas, utilizando bujurqui, Cichlasoma bimaculatum, como presa. Folia Amazónica, 4(1): 133-144.

Barrera, F.; Almeyra, B. 2010. Efecto de la densidad de siembra en el crecimiento de juveniles de Paiche Arapaima gigas en estanques de la estación pesquera Ahuashiyacu.

Brauner, C.J.; Val, A.L. 1996. The interaction between $\mathrm{O}_{2}$ and $\mathrm{CO}_{2}$ exchange in the obligate air breather, Arapaima gigas. And the facultative air breather, Lipossarcus pardalis. Physiology and Biochemistry of the Fishes of the Amazon, 101110.

Castello, L.; Stewart, J. 2010. Assessing CITES nondetriment findings procedures for Arapaima in Brazil. Journal of Applied Ichthyology, 26 (1): 49-56.

Campbell, T. 2004. Hematology of lower vertebrates. In 55th Annual Meeting of the American College of Veterinary Pathologists \& 39th Annual Meeting of the American Society of
Clinical Pathology (ACVP and ASVCP, eds). Ithaca, NY: International Veterinary Information Service. Disponible en http://www.ivis.org

Cavero, B.A.S.; Pereira-Filho, M.; Roubach, R.; Ituassú, D.R.; Gandra, A.L.; Crescêncio, R. 2003a. Biomassa sustentável de juvenis de pirarucu em tanques-rede de pequeno volume. Pesquisa Agropecuária Brasileira, 38 (6): 723728.

Cavero, B.A.S.; Pereira-Filho, M.; Roubach, R.; Ituassú, R.; Gandra, A.L.; Crescêncio, R. 2003b. Efeito da densidade de estocagem na homogeneidade do crescimento de juvenis de pirarucu em ambiente confinado. Pesquisa Agropecuária Brasileira, 38 (1): 103-107.

Cavero, B.A.S.; Pereira-Filho, M.; Bordinhon, A.M.; Da Fonseca, F.A.L.; Ituassú, D.R.; Roubach, R.; Ono, E.A. 2004. Notas Científicas Tolerância de juvenis de pirarucu ao aumento da concentração de amônia em ambiente confinado. Pesquisa Agropecuária Brasileira, 39 (5): 513516.

Del Risco, M.; Velásquez, J.; Sandoval, M.; PadillaPérez, P.P.; Mori. L.; Chu-Koo, F.W. 2008. Efecto de tres niveles de proteína dietaria en el crecimiento de juveniles de paiche, Arapaima gigas (Schinz, 1822). Folia amazónica, 17 (1-2): 29-37. 
De Medeiros, P. 2014. Dietas prácticas com diferentes níveis de proteína e energía na alimentação de juvenis de pirarucu Arapaima gigas (Schinz, 1822) durante a engorda em tanque-rede. Dissertação (Mestrado). INPA, Manaus. 61pp.

De Oliveira, E.G.; Pinheiro, A.B.; de Oliveira, V.Q.; da Silva, A.R.M.; de Moraes, M.G.; Rocha, I.R.C.B.; de Sousa, R.R.; Costa, F.H.F.; 2012. Effects of stocking density on the performance of juvenile pirarucu Arapaima gigas in cages. Aquaculture 370, 96-101.

Drumond, G.V.F.; de Almeida, A.P.; Tavares-Días, M.; Marcon, J.L.; Affonso, E.G. 2010. Biochemical and hematological characteristics of the pirarucu Arapaima gigas Schinz, 1822 (Arapaimidae) from semi-intensive culture in the Amazon. Acta Amazónica, 40 (3): 591-596.

García, A.; Vargas, G.; Deza, S.; Tello, S.; Duponchelle, F. 2011. Situación actual de la pesca en la Amazonía peruana, con énfasis en Loreto. In: Agudelo, E.; Duponchelle, F. (Eds). Comunicaciones del III Coloquio de la Red de Investigación sobre la Ictiofauna AmazónicaRIIA.p. 46-51.

Goldenfarb, B.; Bowyer, P.; Hall, E.; Brosious, E. 1971. Reproducibility in the hematology laboratory: the microhematocrit determination. American Journal of Clinical Pathology, 56(1), 35-39.

Hawkey, M.; Dennett, B. 1989. Color atlas of comparative veterinary hematology. Veterinary Clinical Pathology, 18(4), 108-108.

Imbiriba, E.P. 2001. Potencial de criacao de pirarucu, Arapaima gigas, em cativeiro. Acta amazónica, 31 (2): 299-316.

Ismiño-Orbe, R. A.; Araujo-Lima, C. A. R. M.; Gomes, L. D. C. 2003. Excreção de amônia por tambaqui (Colossoma macropomum) de acordo com variações na temperatura da água e massa do peixe. Pesquisa Agropecuária Brasileira, 38(10), 1243-1247.

Lüling, k. 1971. Der riesenfisch Arapaima gigas in den flussen und seen Amazoniens. Natus Museum, 101:373-386.

Marinho, R.D.G.B.; Tostes, L.V.; Borges, M.; Yoshioka, E.T.O.; Tavares-Dias, M. 2015. Respostas hematológicas de Arapaima gigas (Pisces: Arapaimidae) parasitados naturalmente por protozoários e metazoários. Biota Amazônia, 5(1): 105-108.

Martins, M.L.; Tavares-Dias, M.; Fujimoto, R.Y.; Onaka, EM.; Nomura, D.T. 2004. Hematological alterations of Leporinus macrocephalus
(Osteichtyes: Anostomidae) naturally infected by Goezia lepirini (Nematoda: Anisakidae) in fish pond. Arquivo Brasileiro de Medicina Veterinaria e Zootecnia, 56 (5): 640-646.

Menezes, G.C.; Tavares-Dias, M.; Ono, E.A.; Andrade, J.I.A.; Brasil, E.M.; Roubach, R.; Urbinati, E.C.; Marcon, J.L.; Affonso, E.G. 2006. The influence of dietary vitamin $\mathrm{C}$ and $\mathrm{E}$ supplementation on the physiological response of pirarucu, Arapaima gigas, in net culture. Comparative Biochemistry and Physiology. Part A, Molecular \& Integrative Physiology, 145: 274-279.

Natt, P.; Herrick, A. 1952. A new blood diluent for counting the erythrocytes and leucocytes of the chicken. Poultry Science, 31(4), 735-738.

Nelson, J. S. 1994. Fishes of the world, 3rd edition. John Willey \& Sons, Inc. New York, NY, 624 pp.

Núñez, J.; Chu-Koo, F.; Berland, M.; Arévalo, L.; Ribeyro, O.; Duponchelle, F.; Renno, J.F. 2011. Reproductive success and fry production of the paiche or pirarucu, Arapaima gigas (Schinz), in the region of Iquitos, Perú. Aquaculture Research, 42: 815-822.

Paredes, D.; Álvarez, C.; Valencia, T. 2015. Caracterización hematológica y bioquímica de juveniles de Arapaima gigas "paiches" bajo condiciones de cultivo en selva alta. Revista Investigación y Amazonía, 3 (2): 67-70.

Pereira-Filho, M.; Cavero, B.A.S.; Roubach, R.; Ituassú, D.R.; Gandra, A.L.; Crescêncio, R. 2003. Pirarucu Arapaima gigas husbandry in ponds. Acta Amazónica, 33(4): 715-718.

Pereira, L.P.F.; Mercante, C.T.J. 2005. A amônia nos sistemas de criação de peixes e seus efeitos sobre a qualidade da água. uma revisão. Boletim do Instituto de Pesca, 31(1): 81-88.

Ranzani-Paiva, M.J.T.R.; Pádua, S.B.; TavaresDías, M.; Egami, M.I. 2013. Métodos para análise hematológica em peixes. Editora da Universidade Estadual de Maringá: EDUEM. 139pp.

Saint-Paul, U.; Bayley, P.B. 1979. A situação da pesca na Amazônia Central. Acta Amazonica, 9 (4): 109-144.

Saint-Paul, U. 1986. Potential for aquaculture of South American freshwater fishes: a review. Aquaculture, 54(3): 205-240.

Serrano, M.; Leguía, P.; Quispe, H.; Casas, V. 2013. Valores hematológicos del paiche Arapaima gigas de la Amazonía peruana. Revista de Investigaciones Veterinarias del Perú, 24(2): 248-251. 
Stickney, R.R. 2000. Encyclopedia of aquaculture. John Wiley e Sons. New York. 1067pp.

Tavares-Días, M.; De Moraes, F. R. 2004. Hematologia de peixes teleósteos. 144pp.

Tavares-Dias, M. 2006. Cytochemical method for staining fish basophils. Journal of fish biology, 69(1): 312-317.

Tavares-Dias, M.; Barcellos, J.F.M.; Marcon, J.L.; Menezes, G.; Ono, E.A.; Affonso, E.G. 2007. Hematological and biochemical parameters for the pirarucu Arapaima gigas Schinz, 1822 (Osteoglossiformes, Arapaimatidae) in net cage culture. Electronic journal of ichthyology, 2: 6168.

Val, A.L.; de Almeida-Val, V.M.F.; Affonso, E.G. 1990. Adaptative features of Amazon fishes: hemoglobins, hematology, intraerythrocytic phosphates and whole blood Bohr effect of Pterygoplichthys multiradiatus (Siluriformes). Comparative Biochemistry and Physiology Part B: Comparative Biochemistry, 97(3): 435-440.
Velásquez, J.; Del Risco, M.; Chu-Koo, F.W.; Alcántara-Bocanegra, F.; Chávez-Veintemilla, C.A.; Padilla-Pérez, P.; Tello-Martín, J.S. 2007. Protocolo de adaptación de alevinos de paiche Arapaima gigas al consumo de alimento artificial en cautiverio. Folia Amazónica, 16 (12): 7-10.

Wintrobe, M. 1934. Variations on the size and haemoglobin content of erythrocytes in the blood various vertebrates. Folia Haematologica, 51: $32-49$

Recibido: 16 de Junio del 2016

Aceptado para publicación: 29 de Julio del 2016 